\title{
Millimeter VLBI Observations of the Gamma-Ray Blazar NRAO 530
}

\author{
Geoffrey C. Bower ${ }^{1}$ \\ Radio Astronomy Lab, University of California, Berkeley, U.S.A.
}

\begin{abstract}
We present here 3 epochs of 3 and 7 millimeter wavelength VLBI observations and 2 epochs of lower frequency VLBA imaging of the gamma-ray blazar NRAO 530. These observations document the evolution of the parsec scale jet in this source during the brightest flare in 3 decades. New jet components were created during the flare and are probably related to an increase in gamma-ray activity. The components travel at superluminal velocities, urther confirming the connection between superluminal sources and gamma-ray blazars. The rapid evolution of the source makes tracking of components difficult. It appears that either components significantly decelerate or that there is rapid cooling and acceleration of elections. We may be identifying structure due to standing shocks. The jet is bent on all scales between $100 \mu$ arcsec to 10 arcsec. The results indicate the ability of $3 \mathrm{~mm}$ wavelength imaging to probe the nuclei of blazars rapidly and reliably.
\end{abstract}

The blazar NRAO 530 has been firmly identified as a high energy gammaray source through observations with EGRET (Mattox et al. 1997). Recently, NRAO 530 underwent its brightest flare in 3 decades. That flare peaked at 3 millimeter wavelength in April 1995. In Bower et al. (1997) we presented a study of the evolution of the flare at radio and gamma-ray wavelengths as well as 3 millimeter VLBI observations obtained near the peak of the flare. We concluded that the gamma-ray and 3 millimeter fluxes were correlated over 4 years and that new VLBI components traveling superluminally were produced during the flare. Here, we consider the evolution of the parsec-scale structure following the peak of the flare.

An $8 \mathrm{GHz}$ image of NRAO 530 obtained with the VLBA in October 1995 shows a jet with a position angle of $13^{\circ}$. This axis is 60 degrees to the east of the base of the kiloparsec-scale jet observed with the VLA (Zhang 1996, private communication). A second VLBA observation at $8 \mathrm{GHz}$ in October 1996 showed a similar structure. In both images, the new flux due to the flare is unresolved in the core. The structure in the jet is consistent with $\beta_{a p p} \approx 2 \pm 1$.

We show in Figure 1 VLBI images of the core of NRAO 530 at 86, 43 and $22 \mathrm{GHz}$ between 1994 and 1996. The $86 \mathrm{GHz}$ images were obtained through observations with the Coordinated Millimeter VLBI Array. The 43 and $22 \mathrm{GHz}$ images were obtained with the VLBA. The $22 \mathrm{GHz}$ images do not include structure seen on larger scales, which includes a jet parallel to the $8 \mathrm{GHz}$ jet. The images show that new structure appeared between the first and second epochs at 86 and $43 \mathrm{GHz}$. The position angle of the jet axis is between 28 and $73^{\circ}$, indicating that the jet bends on scales as small as $100 \mu$ arcsec. A new component is not resolved from the core in the first $22 \mathrm{GHz}$ image. We estimate an apparent velocity of $7.5 \pm 2 c$ for the newly appearing components in the 86 and $43 \mathrm{GHz}$ images.

Registration of components between the second and third epochs is difficult. In the simplest registration, we associate the brightest components with each other. We find an apparent velocity of $2.1 c$, implying a significant deceleration.

${ }^{1}$ Present address: Max-Planck-Institut f. Radioastronomie, Bonn, Germany. 


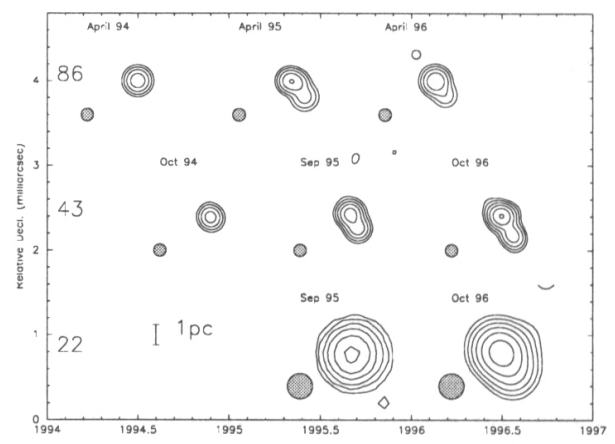

Figure 1. VLBI images of NRAO 530 at 86, 43 and $22 \mathrm{GHz}$ between 1994 and 1996. The images are centered on the ordinate by the date of observation. The convolving beam is shown to the lower left for each image. The contour levels are $0.10,0.25,0.60$, $1.49,3.66$ and $9.00 \mathrm{Jy} /$ beam. A bar in the image shows the scale of $1 \mathrm{pc}=0.24$ mas. The $86 \mathrm{GHz}$ images are from models. The dynamic range of the 43 and $22 \mathrm{GHz}$ images are on the order of 100 and 1000, respectively.

Alternatively, we can assume that the brightest component of the second epoch evolves into the faint component in the north east and that the brightest component of the third epoch is newly emitted from the southwest component. The northeast component is weakly detected in the 86,43 and $22 \mathrm{GHz}$ observations and has a spectrum peaked below $43 \mathrm{GHz}$. The apparent velocities for the outer and inner component are $7.2 c$ and $5.1 c$, consistent with the earlier apparent velocity. This registration requires significant cooling of the electrons in the outer component. This component at $43 \mathrm{GHz}$ declines from 7.4 to 0.2 Jy, implying a magnetic field on the order of $0.5 \mathrm{G}$ if the losses are due to synchrotron cooling. A significant acceleration of electrons must occur in the inner component, producing the new $11.5 \mathrm{Jy}$ component at $43 \mathrm{GHz}$. The rising and declining fluxes must occur on the same timescales to insure the stable observed $90 \mathrm{GHz}$ flux.

A more consistent explanation may involve standing shocks at the location of the two brightest components. Because the initial rise in the total flux does not indicate the creation of a new component in this model, the apparent velocities calculated for the first epoch do not apply. The faint components seen in the third epochs represent weaker traveling shocks. This model is similar to the phenomena seen in 3C 454.3 at $43 \mathrm{GHz}$ (Marscher, these proceedings, p. 25).

Acknowledgments. The National Radio Astronomy Observatory is a facility of the National Science Foundation, operated under a cooperative agreement by Associated Universities, Inc. The Coordinated mm-VLBI Array Project is supported under a grant from the National Science Foundation to the NEROC Haystack Observatory.

\section{References}

Bower, G.C., et al., 1997, ApJ, 484, 118-130.

Mattox, J.R., et al., 1997, ApJ, 481, 95-115. 